\title{
Contrast enhanced coronary MRA at 3.0 T using a 32 channel coil to detect coronary artery stenosis - how does it measure up against 64-slice coronary CTA and X-ray angiography?
}

\author{
Kuncheng Li*1, Qi Yang1, Heng Ma1, Xiaoming $\mathrm{Bi}^{2}$, Lixin Jin ${ }^{3}$, $\mathrm{Han} \mathrm{Li}^{1}$, \\ Jing $\mathrm{An}^{4}$, Renate Jerecic ${ }^{3}$ and Debiao $\mathrm{Li}^{5}$
}

\author{
Address: ${ }^{1}$ Xuanwu Hospital, Beijing, PR China, ${ }^{2}$ Siemens Medical Solutions USA, Chicago, IL, USA, ${ }^{3}$ Siemens Ltd. China Healthcare, Shanghai, PR \\ China, ${ }^{4}$ Siemens Mindit Magnetic Resonance Ltd, Shenzhen, PR China and ${ }^{5}$ Northwestern University, Chicago, IL, USA \\ * Corresponding author
}

from I3th Annual SCMR Scientific Sessions

Phoenix, AZ, USA. 21-24 January 2010

Published: 21 January 2010

Journal of Cardiovascular Magnetic Resonance 2010, I2(Suppl I):P52 doi:10.1 I86/I532-429X-I2-SI-P52

This abstract is available from: http://jcmr-online.com/content/I2/SI/P52

(c) 2010 Li et al; licensee BioMed Central Ltd.

\section{Introduction}

3.0 T contrast enhanced whole-heart coronary MRA has shown high sensitivity for the detection of stenoses in patients suspected of coronary artery disease (CAD). Several studies reported that coronary MRA might have similar accuracy in detecting CAD as 64-Slice CTA. However, no direct comparison between both techniques has been performed.

\section{Purpose}

The purpose of this study was to perform a head-to-head comparison of $3.0 \mathrm{~T}$ whole-heart coronary MRA using 32 channel coils in patients with suspected CAD compared to 64-slice CTA and x-ray coronary angiography (CAG).

\section{Materials and methods}

32 patients with suspected CAD who were scheduled for CAG underwent both coronary MRA and coronary CTA. 3.0 T contrast enhanced whole-heart coronary MRA was acquired by using an ECG-triggered, navigator-gated, inversion-recovery prepared, segmented gradient-echo sequence and 32 channel coils was used with GRAPPA factor of 3. Coronary CTA was performed using a 64-Slice CT scanner. For image analysis, standard 15-segment AHA classification system was used. The diagnostic accuracy of MRA and CTA in detecting significant stenoses $(\geq 50 \%$ of vessel lumen) was compared on the per-segment basis using CAG as reference standard.

\section{Results}

MRA and CTA examinations were successfully completed in all 32 patients. The averaged imaging time of MRA was $6.1 \pm 1.2 \mathrm{~min}$. In a total of 419 coronary segments with a reference luminal diameter $\geq 1.5 \mathrm{~mm}$ on QCA, forty-nine $(12 \%)$ on MRA were evaluated as non-assessable and twenty-eight on CTA were non-diagnostic. On the assessable segments basis, 3.0 T whole-heart CMRA correctly identified 45 significant stenoses, and CTA correctly identified 52 significant stenoses. The sensitivity, specificity, PPV and NPV of MRA and CTA for detecting significant stenoses were $92 \%, 95 \%, 73 \%, 99 \%$ and $96 \%, 92 \%, 67 \%$, $99 \%$, respectively, on assessable segments basis.

\section{Conclusion}

Combined with dedicated 32-channel coils, parallel imaging with higher acceleration factors allows significant reduction in imaging time when compared to our previous results. These improvements resulted in substantially improved overall accuracy of MRA in detecting CAD if only assessable segments were considered. Both MRA and CTA have higher negative predictive values and would be the preferred techniques for noninvasive coronary imaging in ruling out CAD.

\section{References}

I. Qi Yang, Kuncheng Li, Li D, et al.: JACC 2009, 54:69-76.

2. Pouleur AC, Gerber BL: Circ Cardiovasc Imaging 2008, I: | | 4- 12 I.

3. Niendorf T, et al.: Magn Reson Med 2006, 56: I67-I 76. 